\title{
Problèmes et nouvelles solutions pour les fondations des bâtiments ou des équipements dans les régions sismiques
}

\author{
Problems and new solutions for foundations of buildings \\ or equipment in seismic regions
}

\author{
Prof. Ing. Emil TOCHKOV \\ Institut Scientifique du Bâtiment*
}

Rev. Franç. Géotech. n 57, pp. 23-36 (octobre 1991)

\section{Résumé}

Certaines solutions nouvelles relatives à la fondation de bâtiments ou d'équipements importants construits dans les régions sismiques sont présentées. En plus de l'observation stricte des exigences à l'égard des charges conventionnelles, on cherche une certaine réduction de l'action sismique sur la surperstructure des bâtiments (vibroisolation).

On propose ainsi le développement d'un nouveau chapitre de la discipline " Fondation des bâtiments ". Un bref commentaire de trois exemples concrets est aussi présenté.

\begin{abstract}
Certain new solutions for the foundations of structures or important equipment built in seismic regions are presented. Beyond the strict observance of requirements with respect to conventional loads, the research aims at reducing the seismic action on the superstructures of buildings (vibroinsulation).

This leads to proposing the development of a new chapter in the discipline of "Building Foundations ». Brief comments are given on three concrete examples.
\end{abstract}




\section{REMARQUES GÉNÉRALES}

Les 10-20 dernières années ont été caractérisées par une intensité sismique élevée enregistrée aussi bien en Bulgarie que dans plusieurs autres pays du monde. Cela imposa la révision de plusieurs cartes et normes macrosismiques dans le sens d'une tendance accrue au renforcement de la sécurité des constructions et surtout de celles ayant des exigences spéciales. Par exemple, à l'heure actuelle, $95 \%$ du territoire de la Bulgarie a une activité sismique de l'ordre de 7 à 9 degrés d'après l'échelle de 12 degrés MSK-64; la plupart des terrains prévus aux fins de la construction ont une force portante basse ou moyenne, $\sigma$ adm $\leq 250 \mathrm{kPa}$. Le déplacement des chantiers du moins pour un pays comme la Bulgarie est pratiquement impossible. D'autre part, on a besoin de plus en plus souvent de construire des édifices et des équipements ayant des exigences parasismiques élevées comme par exemple des centrales nucléaires, des usines ayant des appareillages électroniques, des tours de télévisions, des bâtiments à plusieurs étages, ainsi que ceux qui relèvent du groupe « $A$ » (1er groupe) conformément aux normes bulgares [3].

D'habitude, pour ce type de bâtiments ou d'équipements, une division en sections microsismiques est prévue, mais franchement parlant les conclusions qu'on peut en tirer sont d'une précision réelle de $\pm 1 / 2$ à 1 degré. En ce qui concerne la plupart de ces bâtiments, il est pratiquement impossible d'apporter des modifications importantes à leur superstructure; par exemple les réacteurs nucléaires soviétiques VVER-1000 sont calculés pour le $8^{e}$ degré sismique (référencé d'après MSK-64), c'est-à-dire jusqu'à une accélération sismique du terrain $\leq 0,2 \mathrm{~g}$ ( $\mathrm{g}=$ accélération de la pesanteur).

La triste expérience des 15 dernières années lorsque notre pays subit quelques tremblements de terre nous a montré que c'est une tâche difficile, lente et coûteuse que de réparer les constructions ordinaires touchées par les actions sismiques. On peut imaginer ce problème rapporté aux édifices spéciaux mentionnés ci-dessus !

Une méthode rationnelle visant à réduire l'action sismique sur les bâtiments consiste indiscutablement à agir sur le mode de leur fondation, étant donné que c'est dans la jonction bâtiment-sol que se produit la transmission de l'énergie sismique. En pratique, l'ingénieur du projet peut $y$ apporter les modifications les plus importantes.

La vibroisolation des fondations soumises à des charges dynamiques des machines est un procédé appliqué depuis longtemps dans le monde entier [7]. Signalons par exemple les réalisations dans ce sens faites par la société allemande spécialisée GERB [1], qui se sert à cet effet de ressorts spirales en acier et d'amortisseurs à viscosité liquide. Il a été proposé de placer des réacteurs nucléaires de manière analogue (fig. 1). Tout cela est bien connu et il y a dans le monde plus de 50 brevets d'invention de vibroisolateurs et d'amortisseurs identiques, mais ils sont appliqués avant tout à des fondations isolées, à des machines ou à des appareils spécifiques tandis que les appli-

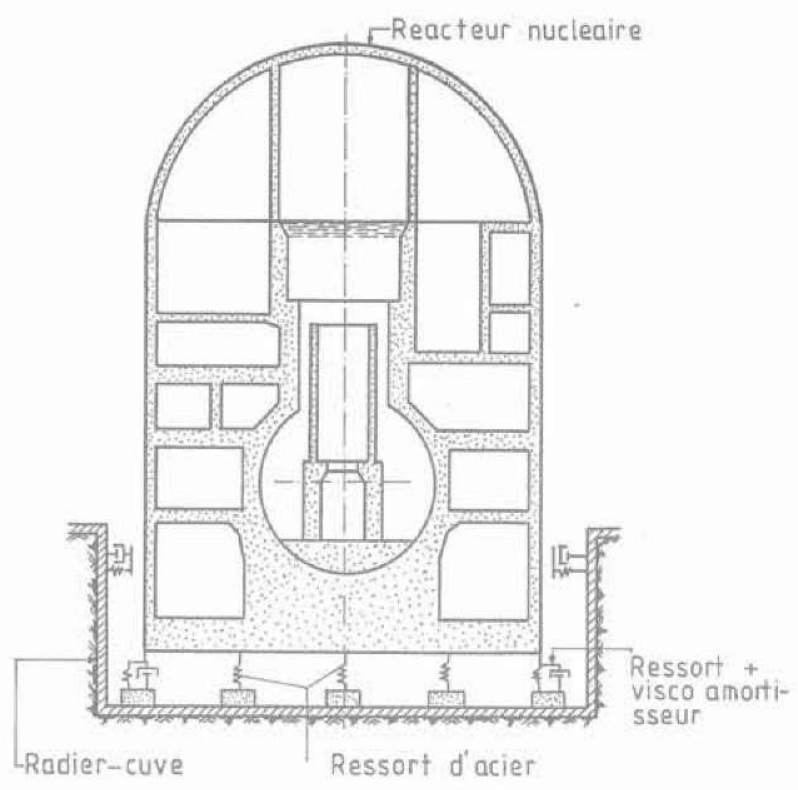

Fig. 1, - Réacteur nucléaire sur des ressorts et amortisseurs (schémas).

Fig. 1, - Nuclear reactor on springs and damping devices (diagram).

cations sont très restreintes en ce qui concerne les bâtiments entiers.

Les raisons en sont les suivantes

- le prix prohibitif des ressorts et des amortisseurs (par exemple le réacteur nucléaire VVER a un poids de $2400000 \mathrm{kN}$ !) ;

- le danger réel de résonance possible dans le cas d'une pareille exécution, étant donné que la période dominante propre des oscillations sismiques $\mathrm{T}_{1}$ varie normalement de 0,3 à $1,5 \mathrm{~s}$ et malheureusement, c'est dans cet intervalle que se trouve la première période propre $\mathrm{T}$ des bâtiments ;

- le remplacement difficile des ressorts et des amortissements ;

- les exigences à l'égard de la stabilité de l'édifice dans les conditions de charge d'exploitation normales.

\section{FONDATION COMBINÉE}

En tant que technologie d'exécution, une méthode, connue depuis longtemps, consiste à utiliser des pieux battus d'une section de $30 / 30$ à $40 / 40 \mathrm{~cm}$, de 8 à $16 \mathrm{~m}$ de longueur disposés à une distance axiale $\mathrm{L}_{\mathrm{o}}$ $=(4,5-6,0) \mathrm{d}$ et un radier en béton armé commun placé sur eux. Les efforts au niveau de la fondation $\Sigma G, \Sigma H$ et $\Sigma M$ sont assumés en commun par les pieux et le radier (fig. 2). Ce dernier est calculé comme un radier sur un lit élastique hétérogène, conformément à un programme établi à cet effet, en prenant compte éventuellement de la rigidité de la superstructure. Toutes les exigences relatives aux normes nationales respectives d'élaboration des projets de fondations superficielles et sur pieux sont respectées. Les avantages de cette solution sont bien évidents : 


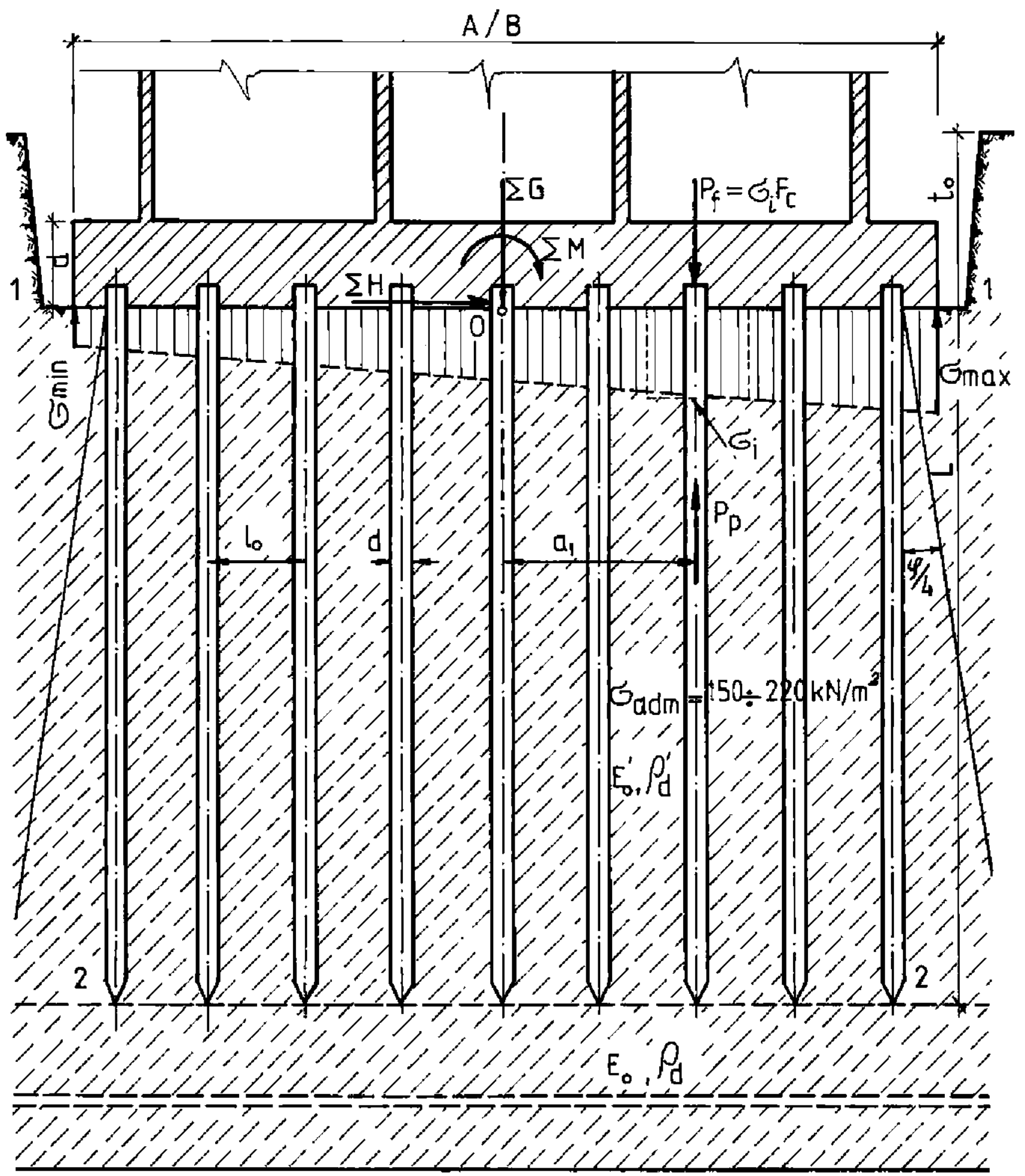

Fig. 2. - Fondation combinée (schéma de calcul).

Fig. 2. - Combirred foundation taliagramt. 
- on épargne des pieux en nombre et en longueur ;

- le battage des pieux est facilité ;

- Les pieux battus compactent le sol ;

- le radier de fondation et les pieux, respectivement projetés, réduisent dans une certaine mesure l'action sismique sur l'édifice [4] ;

- la méthode est simple et adaptable.

En Bulgarie, cette méthode a trouvé une large application dans les cas de fondation de bâtiments lourds sur des sols sableux et limoneux d'une force portante de $\sigma$ adm $\leq 250 \mathrm{kPa}$ et dans les régions sismiques de 8 à 9 degrés. La figure 3 montre un tel exemple: une batterie pour des silos de grains de dimensions de $78 / 48 \mathrm{~m}$ et une charge totale $\mathrm{V}=1,5.10^{6} \mathrm{kN}$ est fondée sur un radier et des pieux battus en béton armé de dimensions $35 / 35 \mathrm{~cm}$ et $\mathrm{L}=12 \mathrm{~m}$, posés à une distance axiale $\mathrm{L}_{0}=1,85 \mathrm{~m}(5,3 \mathrm{~d})$. Ils pénètrent dans un limon lacustre de $\sigma$ adm $=220$ $\mathrm{kPa}, \mathrm{E}_{3}=18 \mathrm{MPa}$, sans atteindre la couche d'assise (argile oligocène). L'intérêt économique, comparé à la solution classique de pieux appuyés en pointe sur l'argile oligocène, est bien évident.

Du point de vue sismique, l'effet favorable de la fondation combinée est dû au radier commun et au sol compacté grâce aux pieux. Dans ce cas, nous avons accepté, tout prudemment, une réduction de l'action sismique sur le bâtiment de l'ordre de 25 à $30 \%$.

\section{DOUBLE RADIER AVEC SEMELLE AMORTISSANTE ET JOINTS DE GLISSEMENT}

Cette solution est présentée, de manière schématique, sur la figure 4. Le radier supérieur (1) est posé au moyen de deux couches préventives en béton maigre (2) sur un joint artificiel (3) qui représente une couche vibroisolante à bas coefficient de frottement $\mu \leq 0,15$ à 0,20 .

Ensuite vient une semelle amortissante en gravier (4) et un deuxième joint de glissement, s'il est nécessaire. La seconde semelle amortissante (5) est en pierre broyée d'une certaine granulométrie et elle est posée sur le radier-cuve inférieur (6). Les deux joints (3) et le radier supérieur (1) sont séparés du radier inférieur (6) par un remplissage de matière élastique molle (7). Le remblai (8) sous le trottoir (9) est en sable compact moyen. Selon chaque cas particulier le nombre des joints glissants est de 1 à 3 ; ils sont épais de 10 à $30 \mathrm{~mm}$ et peuvent être réalisés à partir des matériaux suivants résistant à l'humidité, fluage et usure :

- quelques feuilles en PVC ; feutre imprégné ; feuilles en caoutchouc ; liège bituminé ; toiles imperméables de coefficient de frottement $\mu \leq 0,15$ à 0,20 ; élastomères poreux thixotropiques et autres.

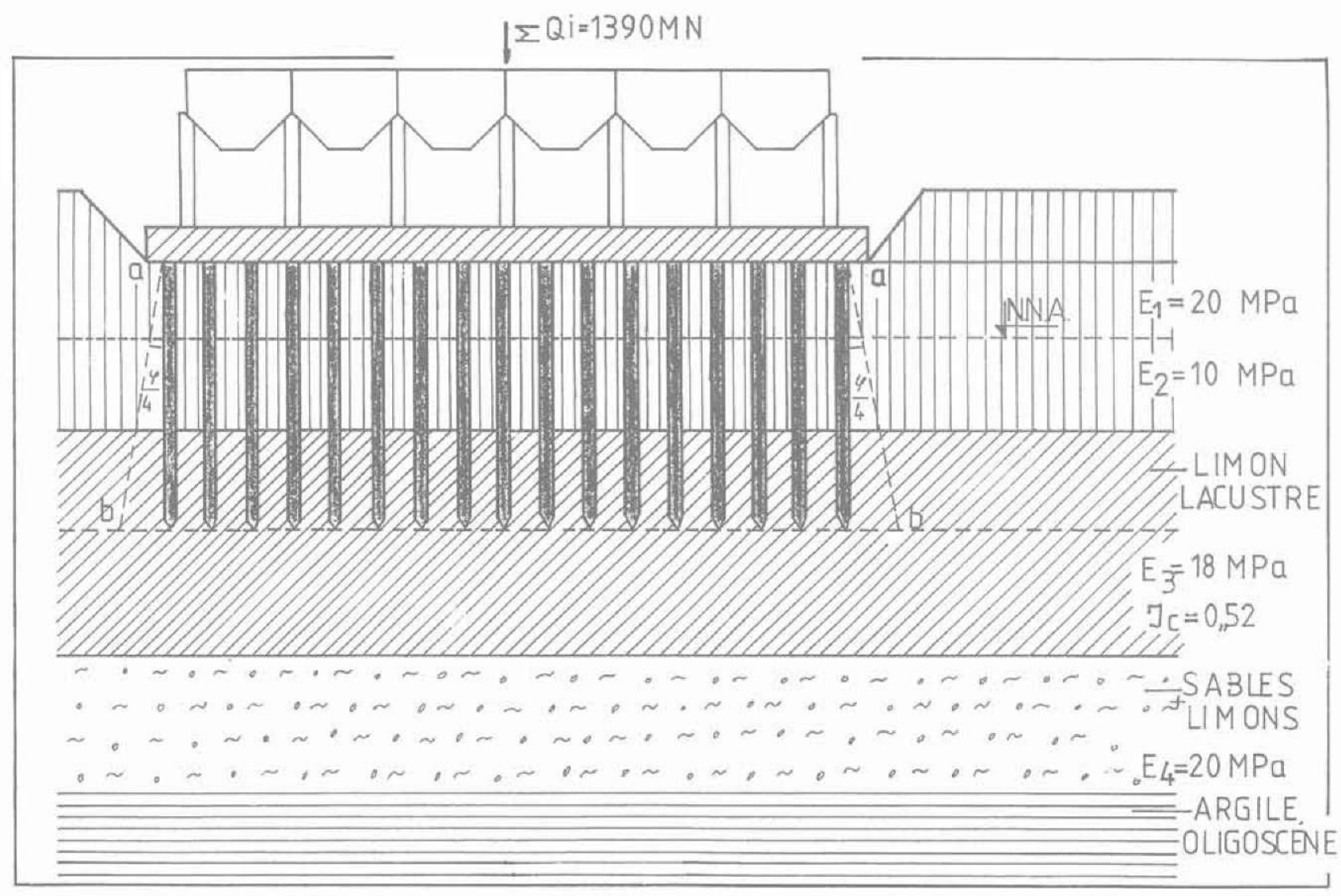

Fig. 3. - Fondation combinée. Exemple de silo de grande hauteur (exemple 1). Fig. 3. - Combined foundation. Example of a high-rise silo (example 1). 

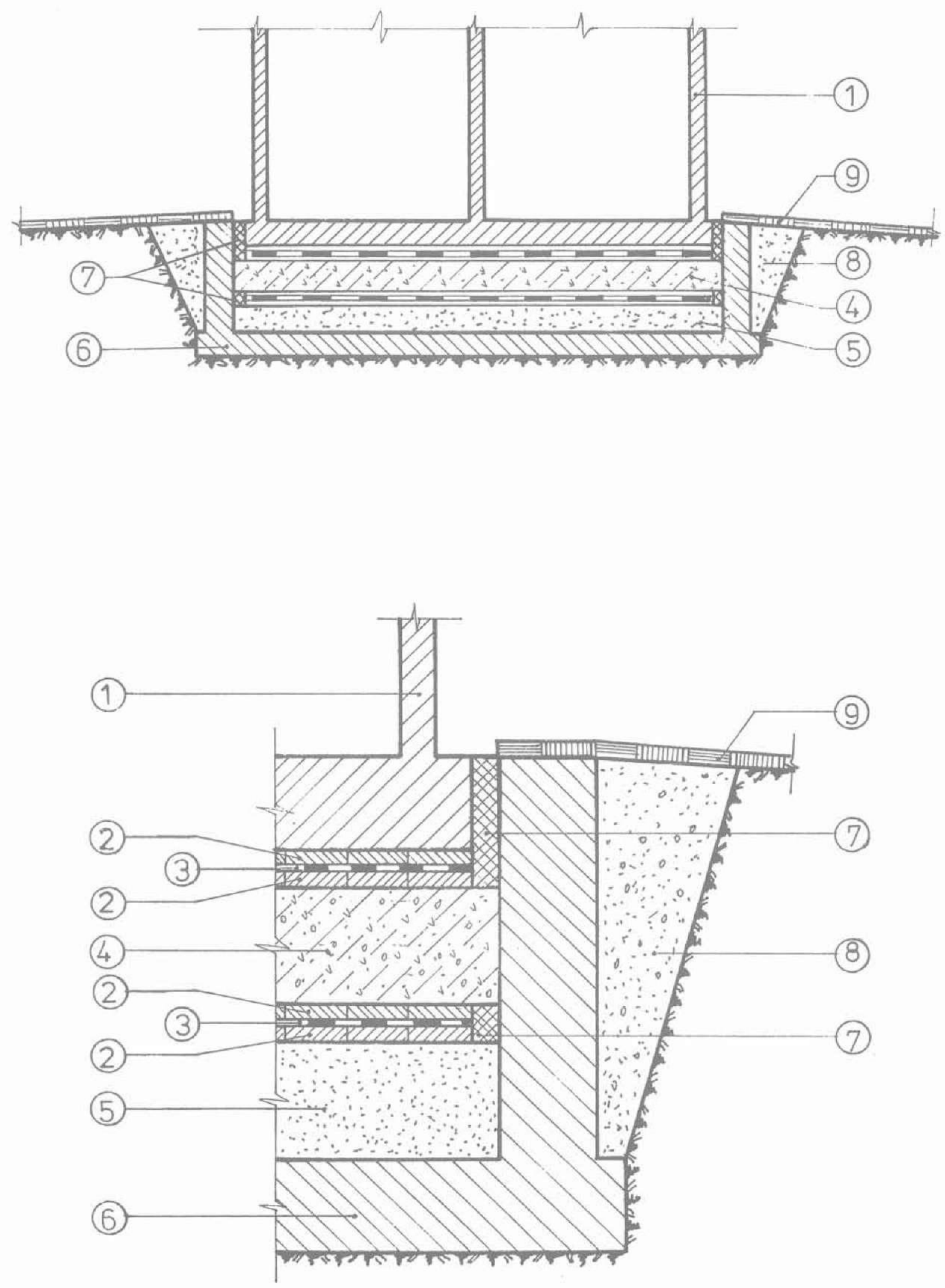

Fig. 4. - Double radier avec couche amortissante + joints glissants (schéma).

Fig. 4. - Double raft with damping layer + sliding joints (diagraml. 
L'exigence principale est la durabilité et un bas coefficient de frottement $\mu \leq 0,20$. Dans des conditions normales, c'est-à-dire, en cas de charge verticale et de vent ou en cas de charge verticale et une secousse faible à modérée, le bâtiment, selon la figure 4 est stable et immobile, étant donné que les forces d'inertie qui sont induites ne sont pas en état de surmonter la friction. Prenons tout simplement le cas élémentaire des oscillations du sol périodiques et purement harmoniques sans amortissement avec un système de construction à un degré de liberté et une masse équivalente $\overline{\mathrm{m}}$ avec un coefficient général respectif de rigidité $\mathrm{C}$ (en $\mathrm{kN} / \mathrm{m})$, c'est-à-dire, avec une période propre du bâtiment (fig. 5) :

$$
T=\frac{2 \pi}{\omega}=2 \pi \sqrt{\frac{\bar{m}}{C}}
$$

La solution des oscillations forcées du système est connue depuis longtemps (par exemple GEIGER, 1958 ) et le point de départ est la condition principale d'équilibre de la masse $\bar{m}$.

$$
\overline{\mathrm{m}} \ddot{\mathrm{x}}+\mathrm{Cx}=\mathrm{Cu}_{1} \cdot \sin \omega_{1} \mathrm{t}
$$

$\mathrm{u}_{1}, \omega_{1}$ et le paramètre $\mathrm{x}$ étant bien visibles sur la figure 5 .

Pour l'oscillation du bâtiment $\mathrm{x}$, on obtient l'expression bien connue:

$$
\begin{gathered}
x=u_{1} \frac{1}{1-\left[\frac{T}{T_{1}}\right]^{2}} \sin \omega_{1} t=\beta u_{1} \sin \omega_{1} t \\
\beta=\left[\frac{1}{1-\left[\frac{T}{T_{1}}\right]^{2}}\right]
\end{gathered}
$$

$\beta$ est le coefficient dynamique ou appelé souvent, en mécanique, coefficient de réponse de la construction.

Au cas où $T=T_{1}$ et $\beta=\infty$, il $\mathrm{y}$ a résonance mais réellement, en conformité avec les normes parasismiques dans le monde entier, on prescrit selon les courbes spectrales $\beta=2,0-3,0[6]$.

Les raisons en sont bien évidentes :

- la loi, de loin plus complexe, de l'oscillation du sol ;

- les forces amortissantes dans la construction ;

- les forces amortissantes dans le sol;

- l'influence aussi bien des oscillations propres de l'édifice et autres [6].

Dans ce cas, l'énergie cinétique de la masse $\bar{m}$, ayant acquis au moment initial la vitesse de l'action sismique $V_{1} \mathrm{~cm} / \mathrm{s}$, se dépense pour la tension du bâtiment (flexion et cisaillement). Admettons maintenant le cas d'un séisme fort et la possibilité d'un glissement de construction sur les joints (fig. 4). Alors la condition d'équilibre (2) se transforme en un mouvement uniformement retardé :

$$
\begin{aligned}
& \overline{\mathrm{m}} \ddot{\mathrm{x}}=-\mu \overline{\mathrm{Q}} \\
& \ddot{\mathrm{x}}=-\mu \mathrm{g} \\
& \mathrm{x}=-\frac{1}{2} \mu \mathrm{gt}_{2}+\mathrm{C}_{\mathrm{o}} \mathrm{t}+\mathrm{C}_{1}
\end{aligned}
$$

Conditions initiales :

$$
\text { Ayant } \quad \begin{aligned}
\mathrm{t} & =0 ; \mathrm{x}=0 \\
\mathrm{t} & =0 ; \frac{\partial \mathrm{x}}{\partial \mathrm{t}}=\mathrm{V}_{1}
\end{aligned}
$$

sans entrer dans les détails, on obtiendra la valeur du glissement du bâtiment :

$$
\mathrm{x}_{\max }=\mathrm{U}_{\mathrm{o}}=\frac{\mathrm{V}_{1}{ }^{2}}{2 \mu \mathrm{g}}
$$

ou bien un résultat encore plus réel:

$$
\mathrm{U}_{\mathrm{o}}=(0,65-0,80) \frac{\mathrm{V}_{1}{ }^{2}}{2 \mu \mathrm{g}}
$$

ou bien ce n'est que pour cette relation que l'effet sismique sur le bâtiment se réduit à l'aire du coefficient $\mathrm{k}_{1}$ :

$$
\mathrm{k}_{1} \cong \frac{1}{1-\frac{\mathrm{U}_{0}}{\mathrm{U}_{1}}}
$$

Selon les données de l'échelle MSK-64, les vitesses obtenues dans les bâtiments pour le degré sismique 8-9 sont de l'ordre de :

$$
V_{1}=10-40 \mathrm{~cm} / \mathrm{s} \text {. }
$$

Pour un coefficient de frottement $\mu$ dans les joints $\mu=0,15-20$, cela signifie des glissements de l'ordre de :

$$
\mathrm{u}_{\mathrm{o}}=5-15 \mathrm{~mm}
$$

c'est-à-dire, au total $30-50 \%$ des amplitudes maximales du tremblement du sol pour les secousses enregistrées en Bulgarie. Le glissement ne doit pas être élevé brusquement, à notre, avis pour les raisons suivantes :

- perturbation de la stabilité de la construction sous l'influence d'autres actions (vent) ;

- danger de chocs sur les bords de la cuve (fig. 4) ; - risques par rapport aux tuyaux et aux communications entrant et sortant du bâtiment;

- problèmes relatifs à la restauration de l'édifice sous son aspect initial.

En cas de réalisation réussie selon la figure 4, nous nous attendons aussi à un autre effet: la dégradation des oscillations de résonance à cause des glissements dans la fondation, étant donné que dans de telles conditions le schéma classique de la construction, définie comme une console complexe encastrée 


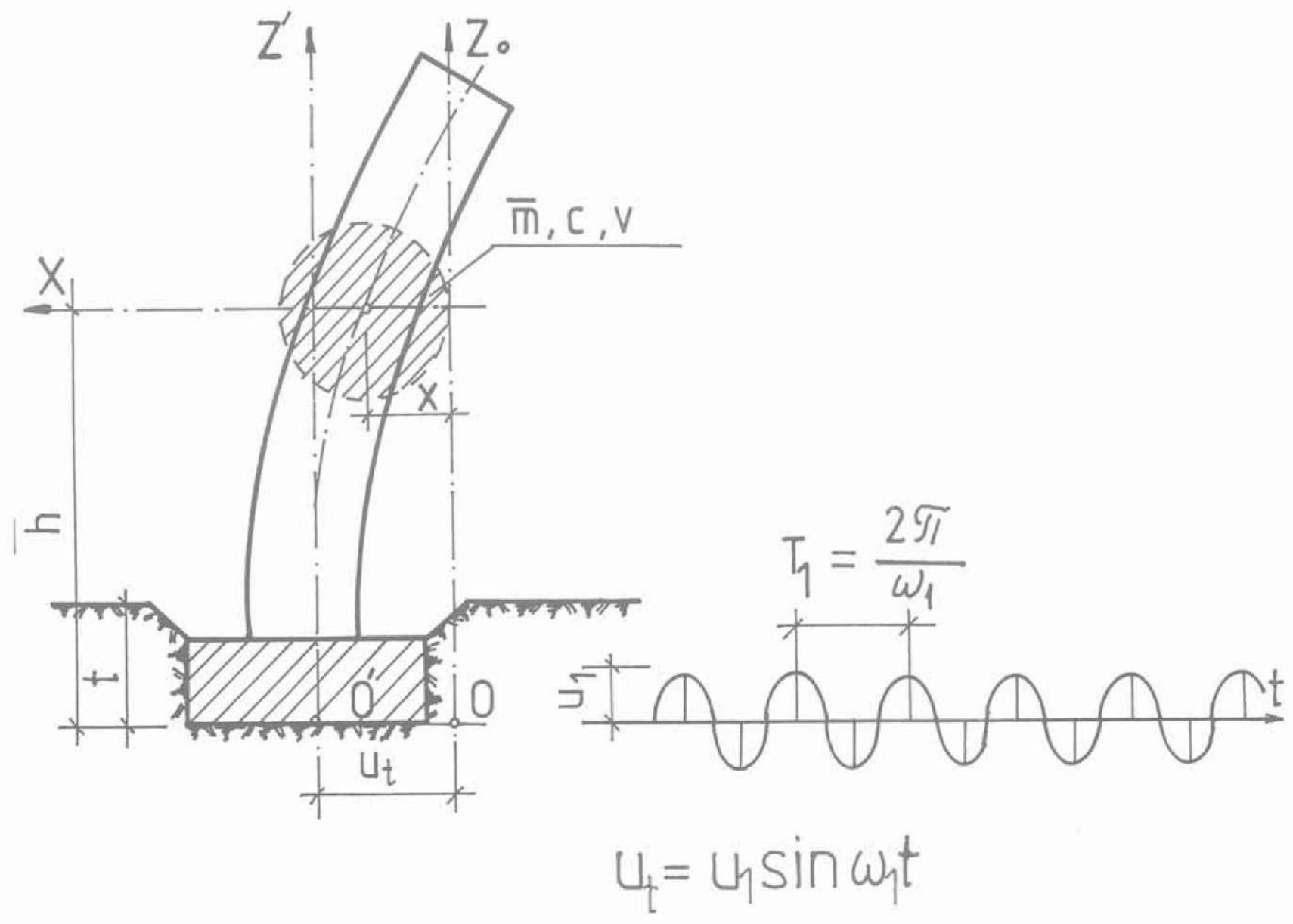

Fig. 5. - Schéma et expressions principales pour l'évaluation du glissement conformément aux options de la figure 4. Fig. 5. - Diagram and main equations for assessing slip in compliance with the options in figure 4.

dans le terrain n'existe pas en réalité. Et cela signifie une diminution brusque du coefficient dynamique bien connu $\beta$. Ou bien dans ce cas, le coefficient commun de vibroisolation sera:

$$
\mathrm{K}_{\mathrm{v}}=\mathrm{K}_{\mathrm{o}} \cdot \mathrm{K}_{1} \cdot \mathrm{K}_{2}
$$

$\mathrm{K}_{\mathrm{o}}$ - de l'effet de la semelle amortissante.

D'après certaines normes mondiales et les vibrations mesurées pour les fondations de machines et d'après les vibrations des moyens de transport:

$$
\mathrm{K}_{\mathrm{o}}=1,25-1,30
$$

$\mathrm{K}_{1}$ - est déterminé selon la formule (6).

$\mathrm{K}_{2} \geqq \beta \min =1,3-1,5$ pour des constructions rigides.

Autrement dit, il s'en suit que selon la méthode de la figure 4 , les édifices et les équipements prévus pour 7-7 1/2 degrés sismiques pourront aussi être réalisés dans des zones de 8 1/2-9 degrés. Pour le moment, en Bulgarie, l'expérience est limitée; elle est avant tout une expérience de laboratoire. A notre avis, le problème crucial est lié aux matériaux pour la vibroisolation. Il n'y a en Bulgarie jusqu'à présent, qu'une décision réglementée, à savoir un radier double muni d'une semelle amortissante en gravier et en sable, en acceptant la vibroisolation

$$
\mathrm{K}_{\mathrm{v}}=1,25-1,30
$$

La figure 6 représente le schéma de fondation d'un réacteur nucléaire à double radier et des pieux lourds en béton armé appuyés en pointe qui ne nécessite pas de nouveaux éclaircissements.

\section{FONDATION DES PIEUX D'APRÈS LA MÉTHODE CHILIENNE}

Dans les cas des édifices lourds et rigides, d'énormes forces d'inertie s'accumulent. Lorsque des pieux bat- 


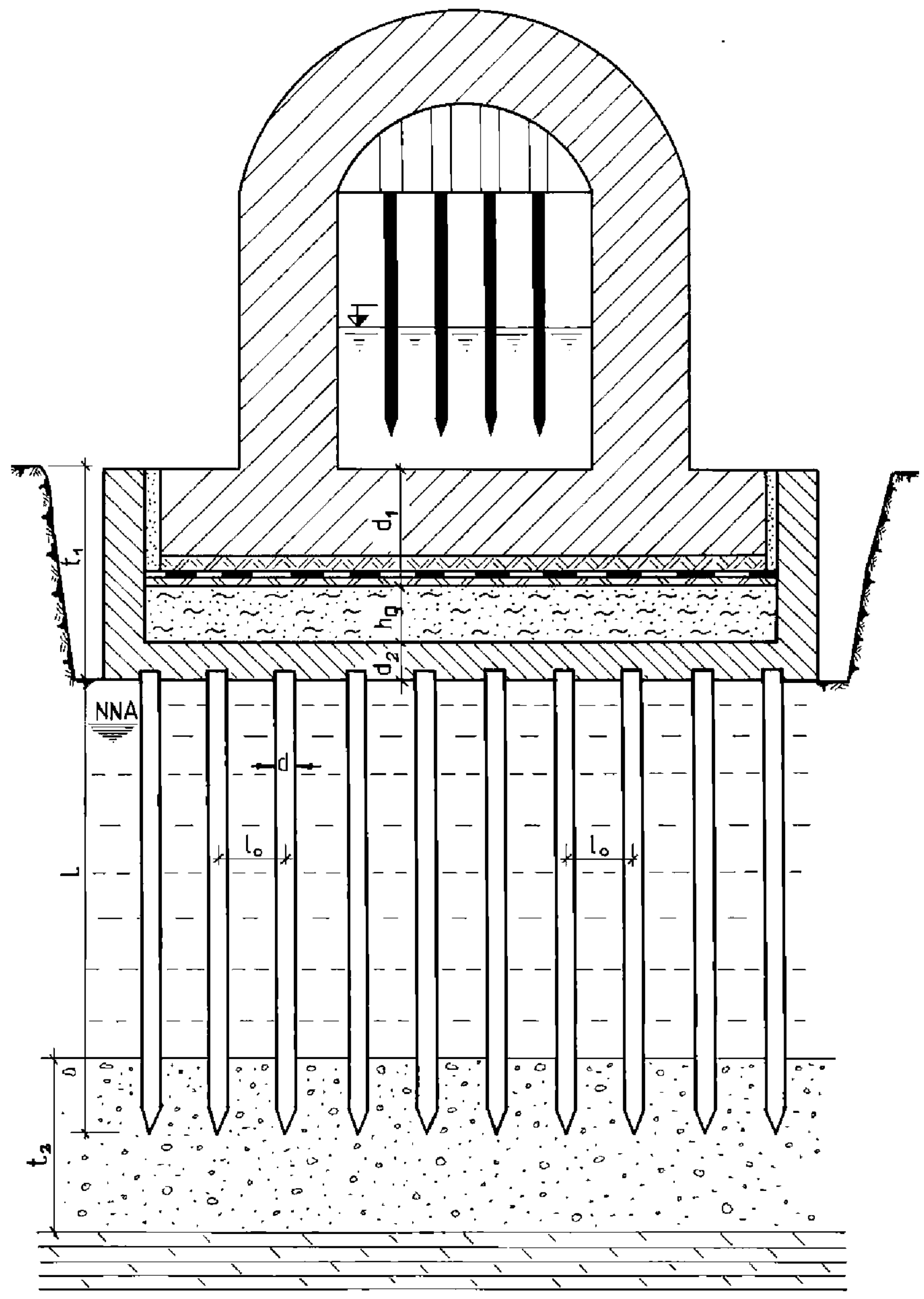

Fig. 6. - Réacteur nucléaire sur radior double et couchs amortissante. Fig. 6. - Nuclear reactor on double raft and damping layer. 
tus sont encastrés dans un radier, le clanger existe qu'ils soient démolis. étant donné qué les efforts transversaux et les moments de flexion dans cette zone deviennent importants. Un mode de fondation permettant de surmonter ce danger est représenté de manière schématique sur la figure 7.

Il a été appliqué avec succès au Chili pour la fondation d'édifices à plusieurs étages dans des régions fortement sismiques: it est réglementé en URSS avec les normes respectives des fondations sur pieux (SNIP - 2.02.03). Il porte là-bas le nom de méthode du VNIIOPS - Moscou. Les dispositions présentées sur la figure 7 sont bier évidentes. Les plaques en béton armé (5) ont 120 à $150 \mathrm{~cm}$ de large et elles assurent une répartition plus régulière de la charge verticale de la construction. Les pieux battus en béton armé $(6)$ sont posés à une distance axiale $L_{0}=(3,5$ a 4,5 ) d et n'assument que les charges verticales de la construction. Les deux semelles amortissantes (3) et (4) ont une épáisseur de $80 \mathrm{a} 100 \mathrm{~cm}$ chacune et sont bien compactes.

Les efforts horizontaux dans le joint de base sont transmis au moyen des forces de frottement, comme c'était le cas des fondations précédentes.

Ce mode de fondation permet les effets suivants : a. les têtes des pieux ne supportent pas les efforts horizontaux et les moments d'encastrement provoqués par la construction ;

b. les pieux rendent le sol compact : s'il est nécessaize on les enfonce dans le terrain à l'aide d'un mouton Diesel et une forte injection d'eau, c'est-à-dire qu'on obtient un massif de sol compacté et des pieux appuyés en pointe (8). Dans ce cas, même la force portante du pieu isolé s'améliore (EC-7, projet 1987. p. 7. 19). Par ailleurs, les pieux en béton sont torte- ment armés, c'est-à-dire qu'ils ont une " ductilité * élevée vis-à-vis des actions sismiques:

c. à l'aide des deux semelles arnortissantes, on obtient aussi une certaine vibroisolation de l'édifice, prise comme on l'avait déjà indiqué, jusqu'à la mesure de

$$
\mathrm{K}_{\mathrm{v}}=1.30-1,40 \text {. }
$$

Un exemple d'application de cette méthode est donné sur la figure 7a. C'est notre proposition de fondation pour les réacteurs nucléaires VVER-1000 No 3 et $\mathrm{N}^{\circ} 4$ de la station nucléaire de la ville de Bélénế en Bulgarie, sur le bord du Danube. La pressiors moyenne d'un réacteur à la base de la fondation à la cote +23 est égale à $550 \mathrm{kPa}$ avec une valeur maximale de $750 \mathrm{kPa}$; force horizontale commune $\Sigma \mathrm{H}=600000 \mathrm{kN}-\frac{1}{4} \cdot \Sigma \mathrm{V}$ : tremblement de terre $\mathrm{a}=0.2 \mathrm{~g}: \beta=2,5$, assurance -10000 années.

La force portante des couches supérieures, en argile et limons est de $\sigma \mathrm{adm}=200-250 \mathrm{kPa}$ : les sables épais fins situés au-dessous ont une portance $\sigma \mathrm{adm}$ $\leqq 150 \mathrm{kPa}$. lls sont lâchés jusqu'à moyennement compacts. Ils sont douteux à la liquéfaction. Les graviers au niveau 8-10 sont compacts, $\sigma$ adm $=500 \mathrm{kPa}$. Le niveau des eaux souterxaines est +18 à +22 $\mathrm{m}, \mathrm{c}^{2}$ est-à-dire que le terrain est saturé. Pour ces raisons. la fondation des réacteurs 1 et 2 est réglée de manière classicue : excavation totale des sols jusqu'au niveau supérieur des graviers. à savoir jusqu'à une profondeur de 10-12 $\mathrm{m}$ et leur substitution par une semelle compacte en gravier fluvial, en couches de $25-35 \mathrm{~cm}$.

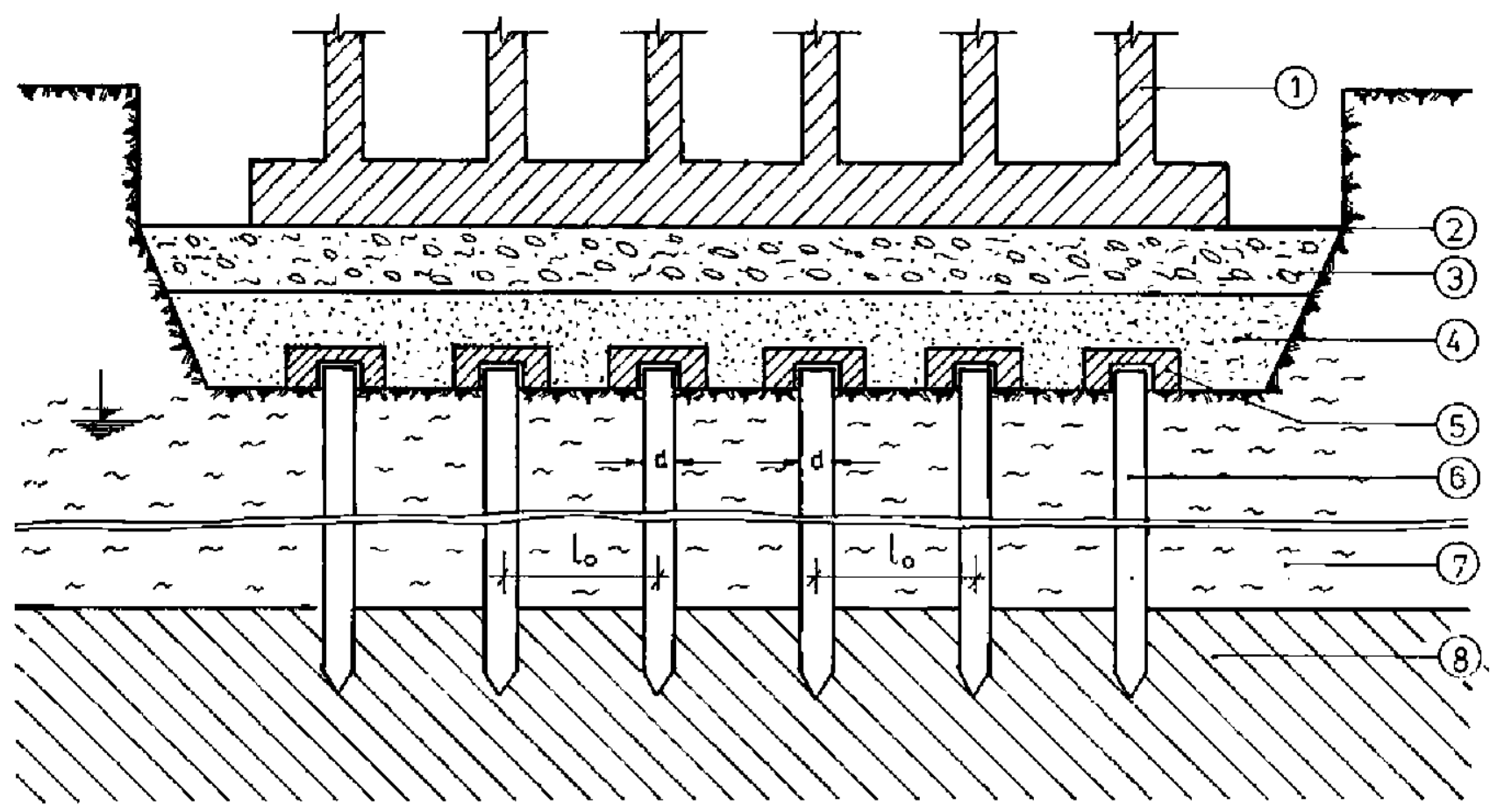

Fig. 7. - Fondation de bátiment lourd sur semelle amortissante at pisux en béton armé (méthode chiliennel.

Fig. 7. - Foundations of heavy bulding on damping footing and reinforced concrete piles (Chilean mathod?. 


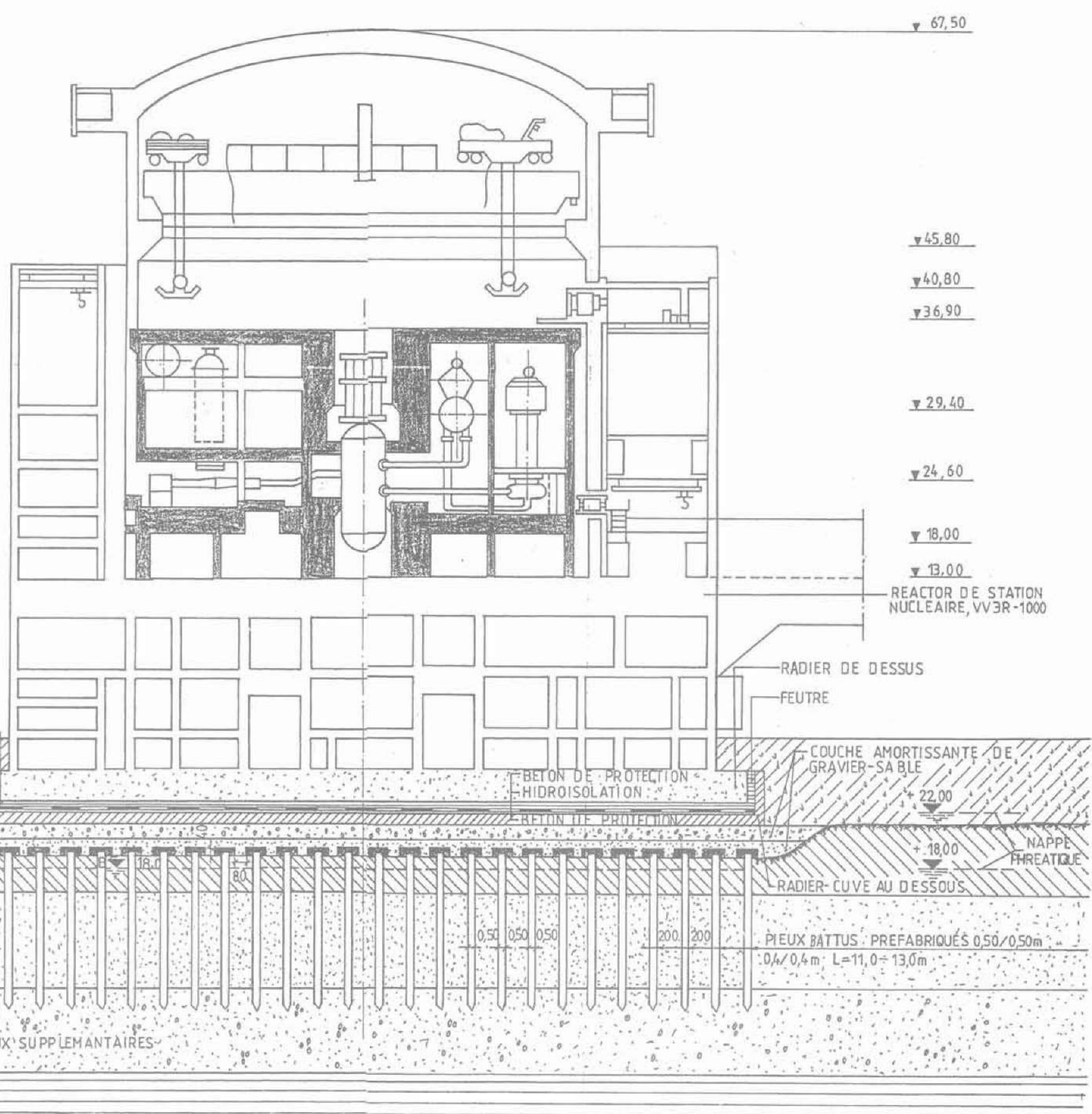

PLAQUES DE BETON ARME $\pm 0,00=28,00$

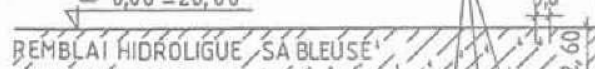

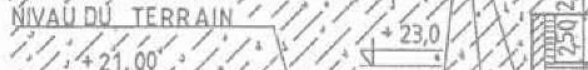

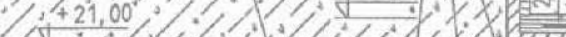

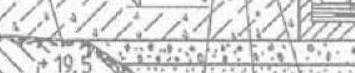

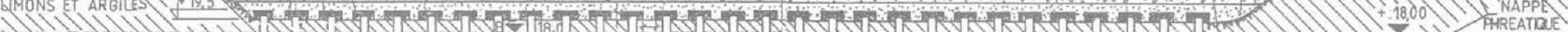
SABLES, FINS: DOUTEUX A : 2 , (1)

GRAVIERS COMPACTS

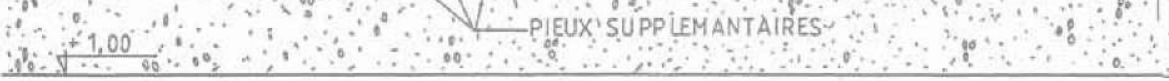

ARGLLE MARNEUSE ET MARNE. 
La fouille est entourée d'une paroi moulée dans le sol qui atteint les marnes. Indépendamment de tout cela, les écoulements dans la fouille sont importants et la réalisation de la semelle se fait en épuisant l'eau continuellement au moyen d'un système spécialement conçu à cet effet.

Les résultats obtenus jusqu'à présent sont bons, mais une pareille réalisation s'avère un processus très lent, difficile à soumettre au contrôle, surtout pendant les mois d'hiver, demandant d'importants travaux de terrassements.

C'est justement pour cette raison que nous avons proposé dans le cas des réacteurs n ${ }^{\circ} 3$ (4) l'adoption de la variante des pieux battus lourds en béton armé de dimensions $50 / 50 \mathrm{~cm}, \mathrm{~L}=12.13 \mathrm{~m}$, ancrés dans les graviers (7a).

Dans le contexte de EC-7 [12], nous nous alignons indiscutablement dans ce cas sur la $3^{e}$ catégorie géotechnique et nous devons avoir pour objectif un contrôle strict du projet. En deux mots, nous appliquons une méthode complexe de prise de solution comportant des initiatives supplémentaires éventuelles au cours de sa mise en cuure pour qu'une sûreté réelle soit atteinte pour un réacteur nucléaire dans une région sismique!

Les initiatives possibles, si cela paraît nécessaire, sont les suivantes:

- la construction d'une barrière antisismique à partir des pieux supplémentaires, liés au-dessus, hors du réacteur. Pour eux, on admet l'apparition des fissurations sous l'action sismique; ils sont les premiers à subir l'attaque des ondes sismiques des couches supérieures du sol le plus faible;

- les principaux pieux de dimensions $50 / 50 \mathrm{~cm}$ sont enfoncés au moyen d'un mouton Diesel du type D-46 (D-55, D-62). Ce travail peut être combiné à une hydrocompaction des sables; des pieux intermédiaires de dimension de $35 / 35 \mathrm{~cm}$ sont aussi possibles ;

- entre le radier supérieur et le radier inférieur, on peut poser une semelle amortissante, par exemple, du type de celle, présentée sur la figure 4.

\section{FONDATION AU MOYEN D'UNE SEMELLE AMORTISSANTE ET D'UN CADRE SOUTERRAIN RIGIDE}

Il s'agit toujours d'un système de fondation articulé, comportant un radier supérieur en forme de grillage, une semelle amortissante et un radier inférieur en forme de cuve porté par un cadre rigide de pieux (fig. 8). La semelle amortissante est du type déjà décrit au paragraphe 2 (avec ou sans joints glissants).

Le cadre souterrain consiste en un radier lisse ou avec nervures et des poteaux appuyés ou encastrés dans une couche d'assise. Ces poteaux sont réalisés avec des pieux forés de gros diamètre, par exemple du type "Benoto », ou avec des diaphragmes de parois moulés, des puits ou autres et ils sont encastrés dans le radier.
Le cadre souterrain est calculé sur la base de la pression sismique directe des couches supérieures faibles du sol pour la force portante et pour avoir une rigidité satisfaisante. On s'efforce que le rapport entre les deux périodes propres, $\mathrm{T}$, de l'édifice posé sur une semelle amortissante et $T_{1}$ du cadre souterrain soient telles que $\frac{T}{T_{1}}>2,0$ pour qu'on puisse obtenir une bonne vibroisolation du bâtiment, du moins pour l'effet des couches supérieures du sol. La période propre de la construction $\mathrm{T}$ ne peut pas varier dans de larges limites, mais pour la réduction de la période $T_{1}$, nous avons des possibilités considérables.

L'étude statique et dynamique d'un tel cadre est une tâche complexe. Une étude numérique à l'aide des ordinateurs s'impose. On s'est servi d'un programme basé sur la méthode des éléments finis (solution bidimensionnelle) qui fournit des déplacements $\Delta \mathrm{X}$ et $\Delta \mathrm{Y}$ et les inclinaisons $\phi$, de même que les efforts tranchants, N, Q et M d'un cadre sur un lit élastique de paramètres volontaires et en toutes conditions de support. Ce cadre est présenté sur la figure 9. La figure $9 \mathrm{a}$ indique les dimensions géométriques, la discrétisation et les moments d'inertie du radier et des poteaux et la figure $9 \mathrm{~b}$ donne le schéma du chargement vertical et horizontal du cadre et de la constante horizontale élastique du lit $\mathrm{C}_{\mathrm{x}}$, accepté selon la loi du triangle conformément aux normes bulgares de fondation sur les pieux et restant du côté de la sécurité.

On a considéré une bande du radier d'une largeur $\mathrm{L}_{\tau}=360 \mathrm{~cm}$ (distance axiale entre les pieux) et d'une hauteur de $h_{\tau}=150 \mathrm{~cm}$. Les pieux sont du type "Benoto ", $d=120 \mathrm{~cm}$, si l'on accepte qu'ils soient appuyés sur la couche d'assise (toujours sur le plan de sécurité).

La poussée sismique horizontale des couches supérieures pour le $8^{e}$ degré $(\mathrm{a}=0,2 \mathrm{~g})$, appartenant à un cadre $\left(\mathrm{L}_{\tau}=360 \mathrm{~cm}\right.$ ) est fixée selon les normes, valables en Bulgarie, et notamment

$$
\mathrm{q}_{\mathrm{x}}=0,144 \mathrm{MN} / \mathrm{m}
$$

est répartie comme cela est représenté à la figure $9 \mathrm{~b}$.

Dans le cas où la première période propre de l'édifice est égale à $\mathrm{T}=0,65 \mathrm{~s}$, on a obtenu pour le cadre souterrain :

a. première période propre: $T_{1}=0,29 \mathrm{~s}$;

b. efforts maximaux au pieu du $1^{\text {er }}$ rang ci-dessus :

- moment de flexion $\mathrm{M}=2,75 \mathrm{MN}$ m ;

- force transversale $\mathrm{Q}=0,37 \mathrm{MN}$

- force axiale $\mathrm{N}=3,36 \mathrm{MN}$ (compression).

Conformément aux normes bulgares pour les projets de constructions en béton armé, on obtient un pourcentage d'armature allant jusqu'à $2,1 \%$ ce qui est acceptable pour un pareil type de pieux (poteaux) lourdement chargés. Ayant en vue les deux effets de la solution :

- la semelle amortissante ; 


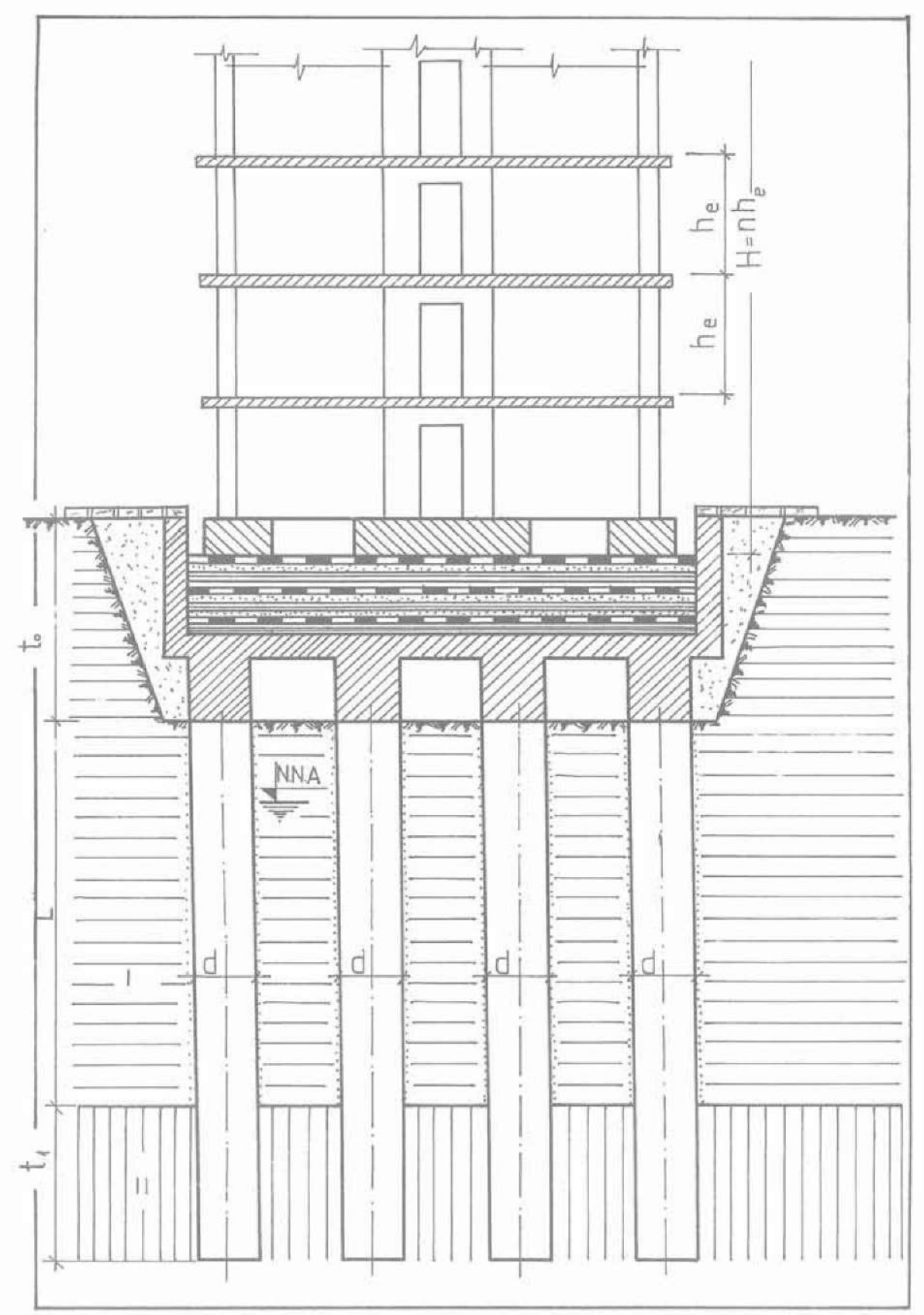

Fig. 8. - Fondation de bâtiment à plusieurs étages sur cadre souterrain rigide (schéma). Fig. 8. - Foundation of a multistorey building on an underground frame (diagram.

- le cadre souterrain, respectivement la relation

$$
\frac{\mathrm{T}}{\mathrm{T}_{1}}=\frac{0,65}{0,29}=2,22
$$

On peut s'attendre à un coefficient de vibroisolation de l'ordre de :

$$
K_{v}=2-3 \text { fois }
$$

c'est-à-dire que la construction projetée pour une intensité sismique de degré 7 à $71 / 2$ pourrait être réalisée dans une région sismique de degré 8 à $81 / 2$ au moyen dudit mode de fondation spéciale. Evidem- ment, ayant en vue les inévitables imprécisions dans l'évaluation de la charge sismique $\mathrm{q}_{\mathrm{x}}$, de la constante $\mathrm{C}_{\mathrm{x}}$ et autres, on doit faire des calculs d'option en tenant compte des dispersions possibles des paramètres géotechniques. Cette difficulté peut être surmontée si l'on dispose de programmes convenables.

Selon les informations de la société GERB [1], il existe des réalisations pareilles. En présence de technique respective moderne de fondation en profondeur (Soletanche - Fr), nous ne voyons pas d'obstacles à la réalisation d'autres projets dans d'autres pays du monde, dans des régions sismiques. 


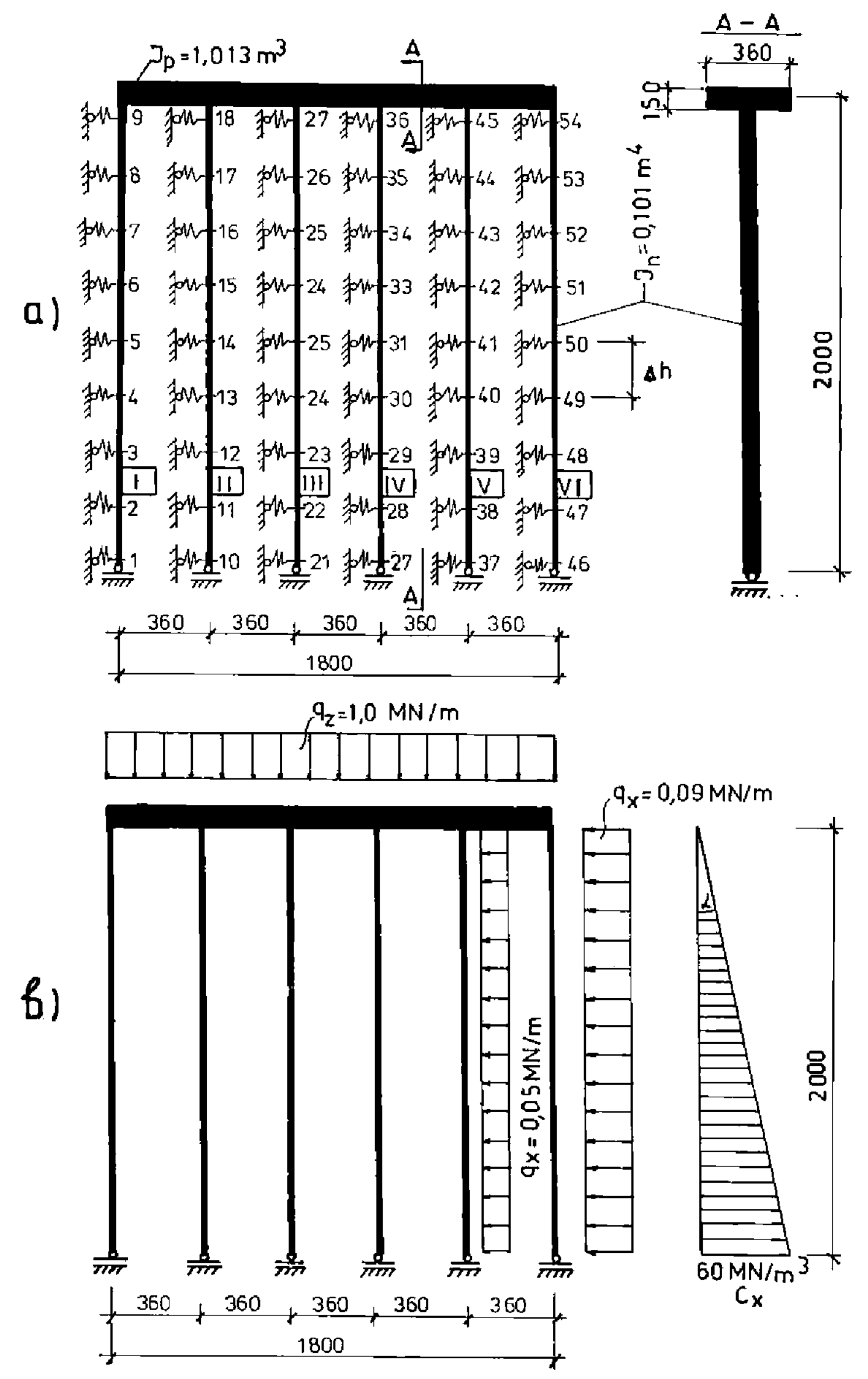

Fig. 9. - Calcul du cadre souterrain an cas d'action sismique horizontale. a. Schéma du cadre. b. Charge et coefficient du lit lexemple 3 .

Fig. 9. - Computation for the underground frame in the case of horizontat seismic action.

a. Diagram of the irame. $b$. Load and coefficient of the footing (example 3). 


\section{CONCLUSION}

Nous venons de décrire certains nouveaux modes de fondation des bâtiments pour lesquels, en plus de l'observation des exigences traditionnelles relatives aux charges verticales, on a cherché une réduction de l'action sismique sur le bâtiment et les équipements. Pour certaines constructions, par exemple les réacteurs nucléaires, posées sur des terrains de $\sigma$ adm $\leqq 250 \mathrm{kPa}$, l'application de pareilles méthodes est, à notre avis, obligatoire.

La vibro-isolation est partielle, mais les solutions données sont réalisables et acceptables en tant que conception aussi bien, pour les charges conventionnelles du bâtiment. D'autres solutions sont aussi possibles. La géotechnique attend de nous un nouveau chapitre, en tant que théorie appliquée, en tant que réalisation mais aussi avec de nouveaux matériaux.

\section{RÉFÉRENCES}

1. Gerb Gessellscharft für Isolieurung mBA \& Co, Essen, Deutschland.

2. Fondation combinée. Prescription du projet. BCA, n 9, 1986, Sofia, Bulgarie.

3. Construction dans les régions sismiques. Normes de projet, 1987, Sofia, Bulgarie.
4. Règles parasismiques, 1969, et annexes, Ed. Eyrolles, 1982, Paris.

5. Snip II-7-81. Construction dans les régions sismiques. 1981, Moscou, URSS.

6. Tentative provisions for the development of seismic regulations for buildings. ATC 03-06-1978, Washington.

7. TOCHKOV Em., Fondations de machine, Ed. Technika, 1969, Sofia, Bulgarie.

8. TOCHKOV Em., Fondations des constructions dans des conditions spéciales, Ed. Technika, 1982, Sofia, Bulgarie.

9. TOCHKOV Em., STAKEV M., Constructions contre le glissement de la terre, Ed. Technika, 1988, Sofia, Bulgarie.

10. TOCHKOV Em., Some new solutions of foundations in seismic regions, 12 ICSMFE, Rio de Janeiro, August, 1989.

11. TOCHKOV Em., Block fundamente unter Stützen und Säulen, H5/1990, Review ÖIAZ, 1990, Wien.

12. Eurocode $n^{\circ} 7$, (EC-7), Geotechnics, Rev. Geotechnik, n 1, 1990, Essen (projet préliminaire, 1989).

13. Eurocode $n^{\circ} 8$, Common unified rules for structures in seismic regions, 1984, BrussellsLuxembourg (Draft). 